\title{
A Method for On-Line Identification and Control Based on Hardware-In-The-Loop Concept
}

\author{
Tarek A. Tutunji and Ashraf I. Saleem \\ Department of Mechatronics Engineering Philadelphia University, Jordan \\ E-mail: ttutunji@philadelphia.edu.jo
}

\begin{abstract}
A method to identify and control a mechatronic system in real time environment is presented in this paper. Acquiring the system's transfer function accurately can be difficult if some parameters are missing. This causes a great difficulty in the system modeling and control. Therefore in this research, a method based on Hardware-in-the-Loop (HIL) concept has been employed to identify and control dynamic systems. Online identification using Auto Regressive Moving Average (ARMA) models is used to identify the transfer function of the system; the controller is tuned on the identified model in a simulation environment and applied to the real system. The advantages of the proposed method are high accuracy in the identified system, simplicity, and low cost. Two case studies, namely, a DC motor and servo-pneumatic system, were used to verify the proposed method.
\end{abstract}

\section{INTRODUCTION}

The HIL is an environment whereby virtual components can be applied on the real system's components. HIL simulation has been applied for designing, developing, and testing hardware and systems. Isermann et al [1] used HIL simulator for diesel engine control design. In their work, HIL was utilized within a structure of a rapid control prototyping system, which allowed fast measurement signal evaluation and rapid prototyping of advanced control engine algorithms. Lin et al [2] developed an HIL simulator for the purpose of a motor cycle power train rapid controller prototyping. In their work, a computer was used to control the operation of the throttle variation and the powder brake torque generation. Hanson et al [3] employed HIL for testing and evaluating the performance of biomedical devices. Their hardware model was interfaced with a computerized model of the cardiovascular system in order to control the beating motion of the hardware heart simulator. In this research, HIL was utilized within a structured algorithm for online identification and control of mechatronic systems.

Researchers developed system identification methods and applied them to many engineering systems. T. Kara and I. Eker [4] presented experimental on-line identification of a three-mass electromechanical system. Tutunji et al. [5], used impulse response data in a recursive gradient algorithm to identify the transfer function of a DC motor and gyroscope. Abdrabbo S. and Tutunji T. [6] presented identification model and sensitivity analysis of hydrostatic transmission system. Yan et al [7] used a recursive prediction error method based on the ARMA model to identify the transfer function of a $\mathrm{CNC}$ milling machine.

In this work, a structured method to identify and control dynamic system's behaviour online was used. HIL concepts were devised to facilitate the realisation of the proposed method. The developed method provides an excellent environment to support design, simulation, and emulation of control systems.

This paper is divided as follows: section 2 presents the HIL and Software-in-the-Loop (SIL) concepts. Section 3 gives the system identification method used. Section 4 details the proposed method. The experimental setup and results are discussed provided in sections 5 and 6 . Finally, the conclusions are summarized in section 7 .

\section{HARDWARE-IN-THE-LOOP CONCEPT}

Generally, classical Mechatronic systems comprise of a controller, actuators, and sensors. The controller generates an output according to the feedback signal from the sensors and sends it to the actuator that performs a certain task. According to the above situation, some components can be substituted by its model and simulated in real time. The simulated components can be run in conjunction with real components under the same environment. This environment is regarded as Hardware-in-the-Loop (HIL). Fig. 1 shows the concept of the employed environment.

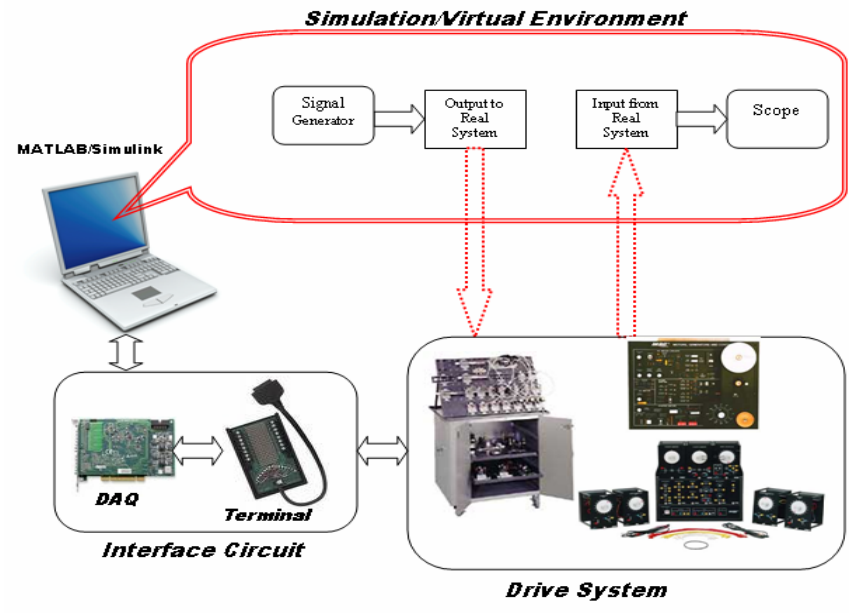

Fig. 1. HIL structure for online identification and control. 
The HIL is an environment whereby virtual components can be applied on the real system's components. Furthermore the HIL gives the capability to monitor the system's behavior by observing the output signals such as speed and position signals. These signals can be stored and used to analyze the system's behavior.

On the other hand, Software-in-the-Loop (SIL) is an environment that allows the control system to be built and synthesized with different virtual components by simply replacing the component according to the control algorithm requirements. In this environment the virtual controller is applied on a simulated model of the real system for optimization and tuning purposes.

\section{SYSTEM IDENTIFICATION MODEL}

In an Auto Regressive Moving Average (ARMA) model, the system to be identified is assumed to have the following linear model[8]

$\hat{y}(k)=\sum_{j=1}^{n} a_{j} y(k-j)+\sum_{i=0}^{m} b_{i} u(k-i)$

where $u(k), y(k)$ and $\hat{y}(k)$ are the input, the original system output, and the predicted model output at the $k^{\text {th }}$ sample.

The goal is to find a linear system model that gives output ( $\hat{y}$ ) equal to real output $(\mathrm{y})$. The least square error between the actual and modeled output is given by

$$
\text { error }=\frac{1}{2} \sum_{k=1}^{K}(\hat{y}(k)-y(k))^{2}
$$

Input-output patterns $(u, y)$ are available. They are used in the above equations to calculate $\hat{y}$. The parameters $a_{j}$ and $b_{i}$ are updated to minimize the least square error as shown in Fig. 2.

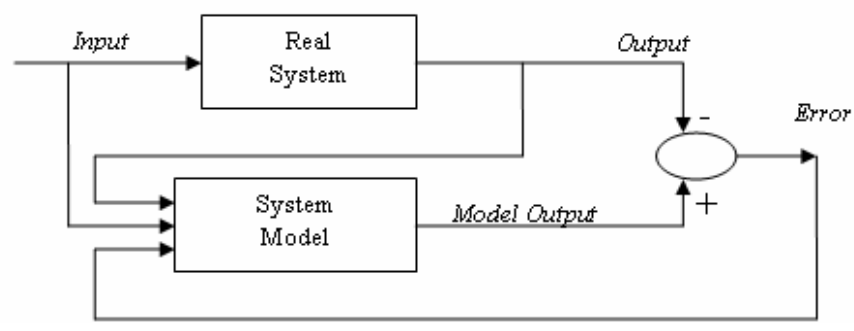

Fig. 2. Prediction Error Model Block Diagram

In vector format the following vectors are defined:

$$
\begin{aligned}
& \Phi^{T}(k)=\left[\begin{array}{llllll}
y(k-1) & \ldots & y(k-n) & u(k) & \ldots & u(k-m)
\end{array}\right] \\
& \theta^{T}=\left[\begin{array}{llllll}
a_{1} & \ldots & a_{n} & b_{0} & \ldots & b_{m}
\end{array}\right]
\end{aligned}
$$

Recursive Least Square (RLS), in vector format, gives the following equations

$\theta=\theta-Q \varepsilon$

$\varepsilon=\Phi^{T} \theta-y$

$Q=P \Phi$

$P=\left\{P-P \Phi \Phi^{T} P /\left(\lambda+\Phi^{T} P \Phi\right)\right\} / \lambda$

Where $\mathrm{P}$ is a positive definite matrix initialized to be cI (I: identity matrix, $100<\mathrm{c}<10,000)$ and $\lambda$ is the forgetting factor $(0.95<\lambda<0.99)$. Equations $(2.4)$ are used in a recursive algorithm where the parameter vector $\theta$ is updated at every iteration until convergence.

Once the parameters are identified, the Z-Transform of the $\operatorname{ARMA}(m, n)$ model is calculated to yield the estimated Transfer function of the model which is given by

$$
\begin{aligned}
& Z\left\{y(k)-\sum_{j=1}^{n} a_{j} y(k-j)\right\}=Z\left\{\sum_{i=0}^{m} b_{i} u(k-i)\right\} \\
& =>H(z)=\frac{Y(z)}{U(z)}=\frac{b_{0}+b_{1} z^{-1}+\ldots+b_{m} z^{-m}}{1-a_{1} z^{-1}-\ldots-a_{n} z^{-n}}
\end{aligned}
$$

\section{ON-LINE IDENTIFICATION AND CONTROL METHOD PROPOSED}

Online identification can be defined as an algorithm that builds a dynamical mathematical model using measured data in a real time environment. The dynamical mathematical model in this perspective is the estimated transfer function of the identified system.

The proposed methodology used in this research follows the following stages (as illustrated in Fig.3):

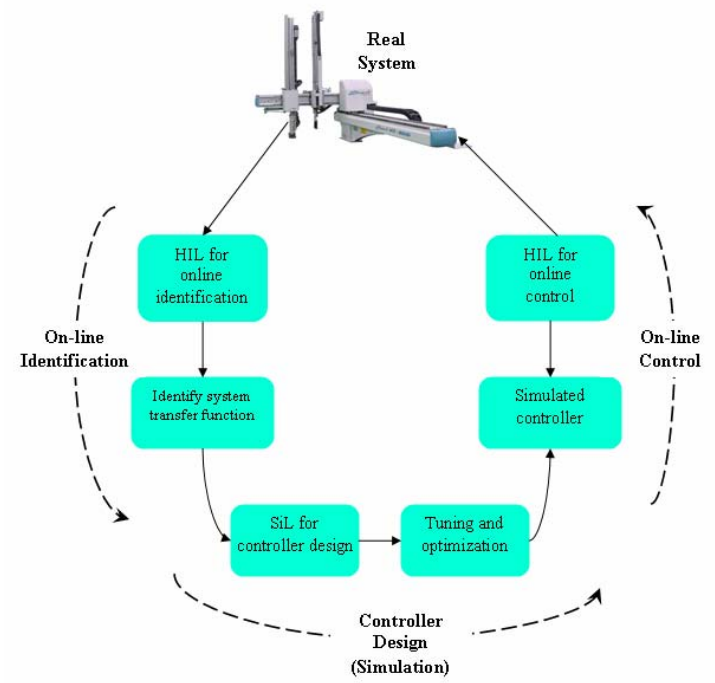

Fig. 3. The proposed method for online identification and control 
Stage 1 on-line identification: In this stage the dynamic system is connected to the computer through data acquisition card. A computer program using Matlab/Simulink is used to stimulate the system by applying an impulse and acquiring its response. The computer algorithm uses these input/output data to identify the system's transfer function using ARMA model and recursive least square algorithm. The above procedure is referred to as HIL identification.

Stage 2 controller design: Once the transfer function is identified, the dynamic system is disconnected and the controller design is performed in a pure simulation environment. The design strategies can vary from PID controllers to intelligent neuro-fuzzy controllers. At this stage the controller is also optimized. This process is referred to as SIL simulation, where a software version of the controller is tested with the simulated plant.

Stage 3 online control: After the controller has been designed and optimized, the dynamic system is reconnected to the computer in order to test the controller and carry out fine tuning online. This is referred to as HIL control.

The advantages of the proposed method include the following: accuracy in the identification and system control, optimizing time resources and minimizing the cost as a result of online identification and offline controller design, increased flexibility of the controller, and user friendly.

\section{CASE STUDY: DC MOTOR}

In order to verify the proposed method and to show its feasibility, an online identification and control was implemented on a DC motor. The experimental test station on which all tests were carried out is schematically shown in Fig. 4 and photo Fig. 5. The motor is driven by a conditioning block which includes chopper circuit, filters, and amplifiers. An optical Tacho-meter is used to measure the position and speed of the rotor. All signals are sent to a computer via National Instrumentation NI DAQ card model 1036E, conditioning terminal. The DAQ card has sampling rate of $200 \mathrm{kS} / \mathrm{s}$, and input voltage range of $\pm 10 \mathrm{~V}$.

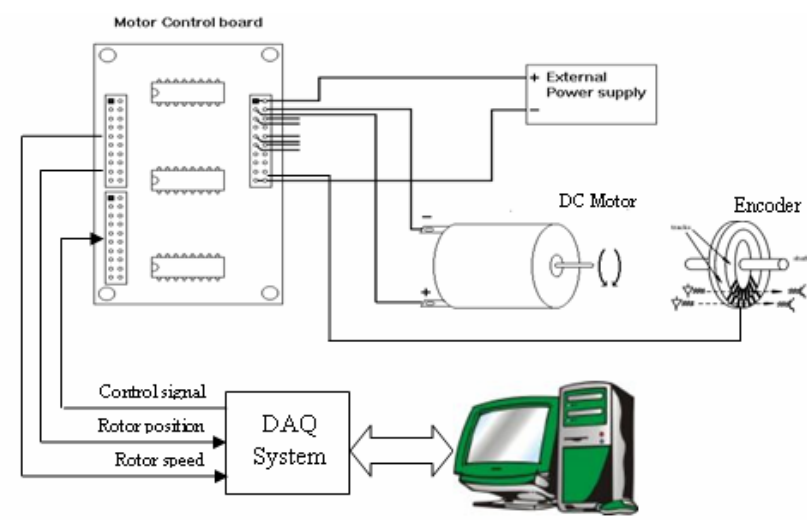

Fig. 4. Experiment setup for DC motor control

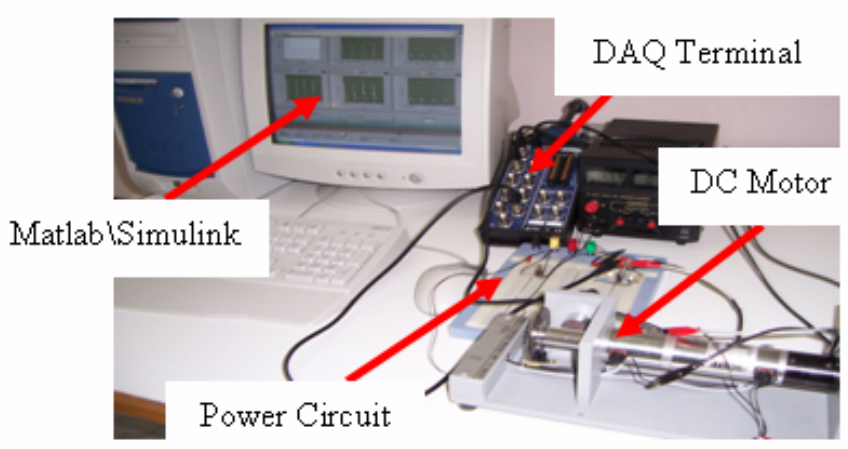

Fig. 5. Photograph of DC motor test set up

In order to investigate the plant characteristics and transfer function, a group of experiments were performed on the test set up shown in Fig. 5.

Online identification using ARMA model was obtained by using the impulse response of the dynamic system. Fig. 6 shows the simulink block diagram of online system identification that has been interfaced to the dynamic system through the DAQ card. Input signal was applied to the DC motor drive via analog output block (DAQ output). The response of the motor (Tacho-meter reading) was measured and sent back the computer through the analog input block (DAQ input). Several trials of different ARMA orders were used ranging from $2^{\text {nd }}$ to $5^{\text {th }}$ order. $4^{\text {th }}$ order model shows the best match between the actual output and the predicted model output.

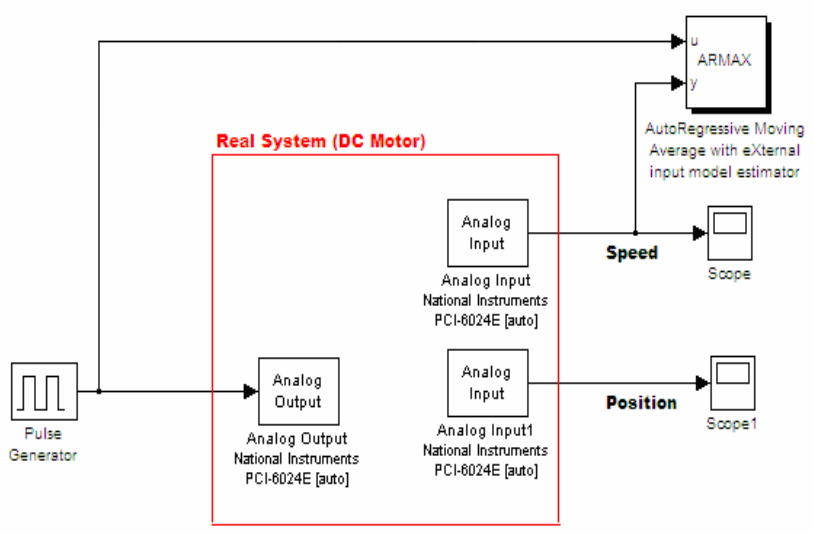

Fig. 6. HIL Simulink block diagram for online identification

Fig. 7 represents, online actual output of an $4^{\text {th }}$ order predicted model with one step prediction. The corresponding transfer function is given below:

$G=\frac{-0.0037451 z^{4}+0.15068 z^{3}-0.06158 z^{2}+0.027998 z}{z^{4}-1.5664 z^{3}+0.96379 z^{2}-0.44964 z+0.16286}$ 


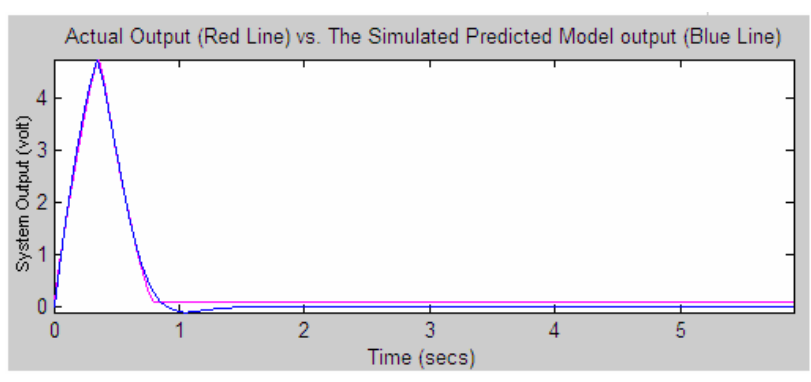

(a)

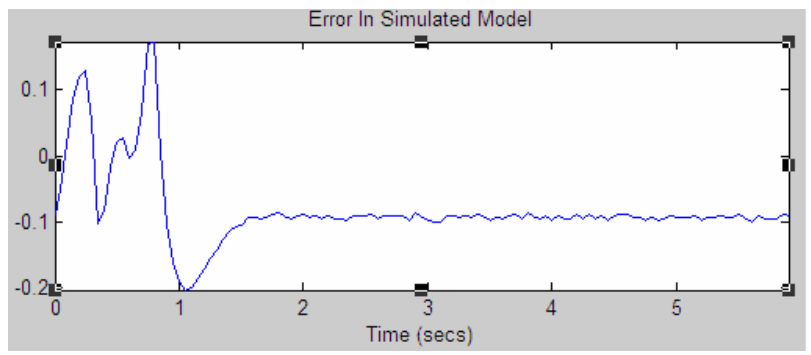

(b)

Fig.7. (a) On line actual output VS $4^{\text {th }}$ order predicted model at one step prediction (Note: $5 \mathrm{~V} \rightarrow 1590 \mathrm{rpm}$ ), and (b) the error between the actual system's output and the predicted model verses time

It is worth noting that the identified transfer functions shown in $\mathrm{Eq}$ (6.1) is not unique since the ARMA model gives an approximation of the original transfer function through a minimization algorithm that converges to a local minimum not a global.

A desired speed profile was prepared to be used as a reference input signal for both simulated and real system. Software block of Proportional-Integral Derivative (PID) controller was designed to control the motor speed. The values for proportional gain $\mathrm{K}_{\mathrm{p}}$, integral gain $\mathrm{K}_{\mathrm{i}}$ and derivative gain $\mathrm{K}_{\mathrm{d}}$ parameters were carried out in SIL environment, as shown in Fig.(8), using Ziegler-Nicholas method [9]. The final parameters used were $\mathrm{K}_{\mathrm{p}}=25, \mathrm{~K}_{\mathrm{i}}=3$, and $\mathrm{K}_{\mathrm{d}}=0.5$

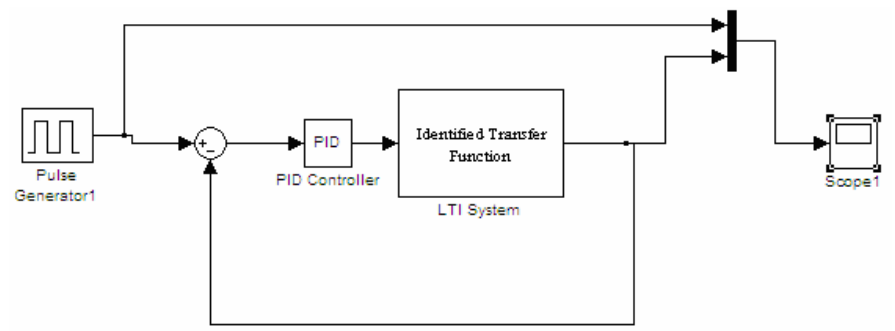

Fig.8. SIL environment for controller design and tuning

After achieving satisfactory system speed response, the optimised controller was applied to the dynamic system through the HIL (see Fig. 9).

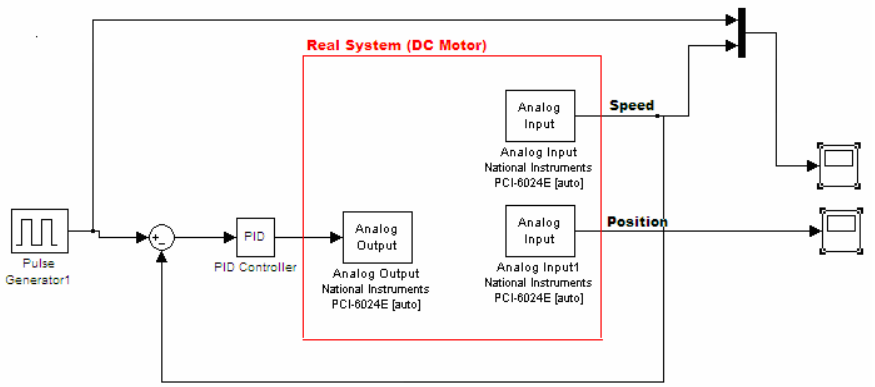

Fig. 9. Simulink block for HIL real time control system

Fig. 10 shows the real and simulated system speed response to the desired speed profile. (Note: $5 \mathrm{~V}$ output is equivalent to $1590 \mathrm{rpm}$ )

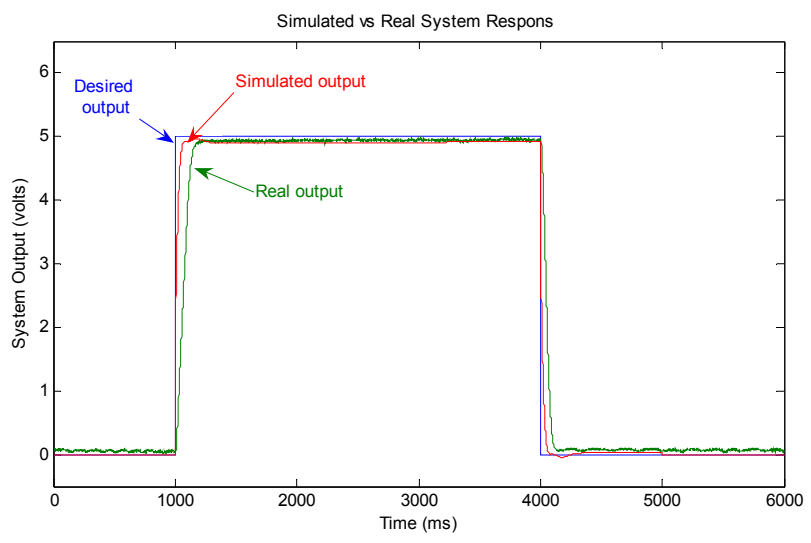

Fig. 10. Simulated vs real system response

\section{Case Study: Pneumatic Servo Drive}

A second case study was performed on a servo-pneumatic system. The experimental test station on which the tests were carried out is shown in Fig.11. The pneumatic unit consisted of pneumatic power supply which included compressor with air conditioning unit, lubricating unit, and manifold. Servopneumatic valve has $1 / 8$ inch port and operating voltage from zero to 10 volts and pneumatic actuator has piston diameter of $27 \mathrm{~mm}$, rod diameter of $8 \mathrm{~mm}$ and stroke length of $100 \mathrm{~mm}$. A rotary potentiometer has resistance range from 2 to $12 \mathrm{~K} \Omega$ with voltage source of 10 Volts, fixed on the cylinder is used for measuring the position of the piston and providing the position feedback. 


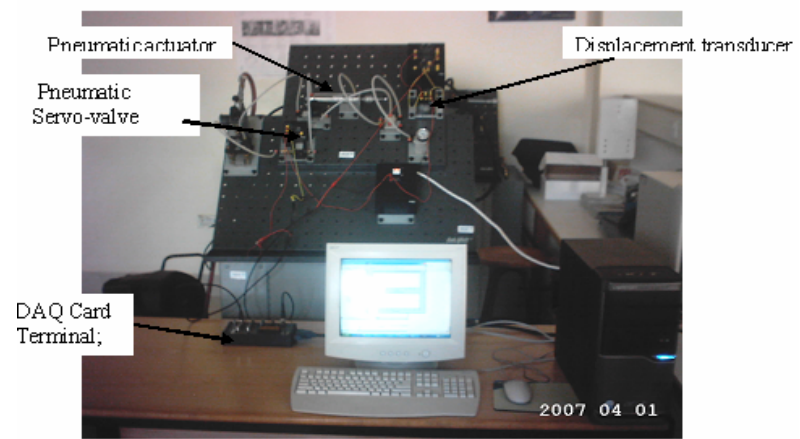

Fig. 11 Photograph of pneumatic test set up

Online identification using ARMA model was obtained by using the impulse response of the real system. A Simulink block diagram was created for online system identification and interfaced to the real system through the DAQ card. Input signal was applied to the servo drive via analog output block (DAQ output). The response of pneumatic actuator (piston displacement) was measured and sent back the computer through the analog input block (DAQ input).

The experiment was performed with 6 bars supply pressure, 2 bars back pressure, and $1 \mathrm{~ms}$ sampling time. Fig. 12 represents, online actual output and $4^{\text {th }}$ order predicted model with one step prediction, square error was $3.22 \mathrm{e}-5 \mathrm{~cm}$ with standard deviation of 0.00571 and the corresponding transfer function was depicted as;

$$
G_{P}=\frac{0.0070699 z^{4}-0.017738 z^{3}+0.019649 z^{2}+0.00027783 z}{z^{4}-1.9827 z^{3}+1.2989 z^{2}-0.43504 z+0.12436}
$$
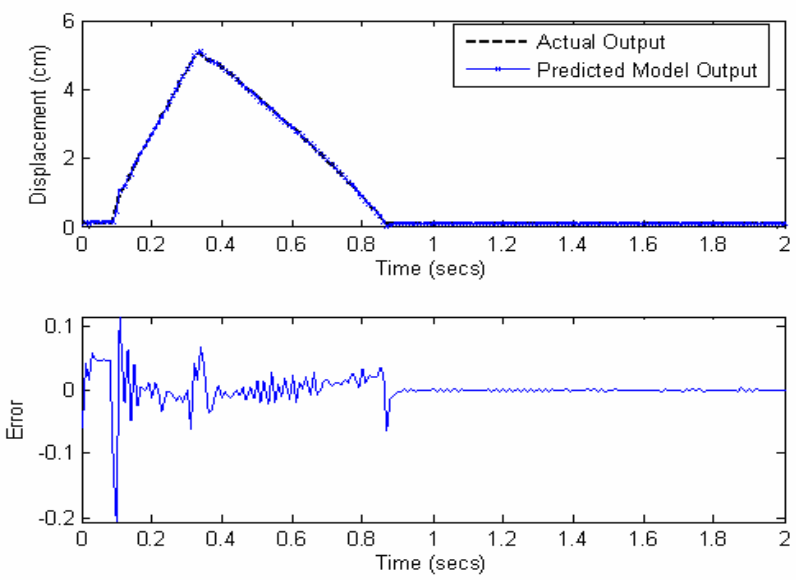

Fig.12 Variation of On line actual output, $4^{\text {th }}$ order predicted model at one step prediction and the error verses time

A desired speed profile was prepared to be used as a reference input signal for both simulated and real systems.
Software block of Proportional-Integral Derivative (PID) servo controller was designed to control the position and speed of the pneumatic actuator. The challenge in PID controller design is to tune the values of the proportional gain $K_{p}$, integral gain $K_{i}$ and derivative gain $K_{d}$. Tuning work is usually performed manually by trying out different tuning parameters combinations on-line until a satisfactory or at least acceptable results are achieved. This method is laborious, time consuming, unsafe and does not always give the best possible solution. This was the motivation to tune the controller parameters under simulation environment (Off-line tuning) using one of the tuning techniques such as Ziegler-Nicholas method to avoid the drawbacks of manual tuning method. Fig. 12 shows the variations of the desired speed profile and resulting simulated speed response at the shown tuning parameters.

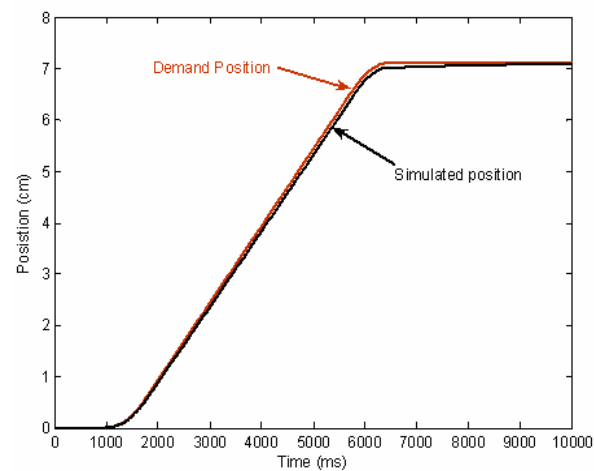

Fig.13 Variations of real and demand displacement verses time at $K p=14, K i=6$ and $K d=0.2$

After achieving satisfactory results from off-line tuning, the optimised controller(s) was applied to the real system through the proposed MRE. The results (as shown in Fig.14) insure much closed tracking of speed profile with good time response characteristics of the real system.

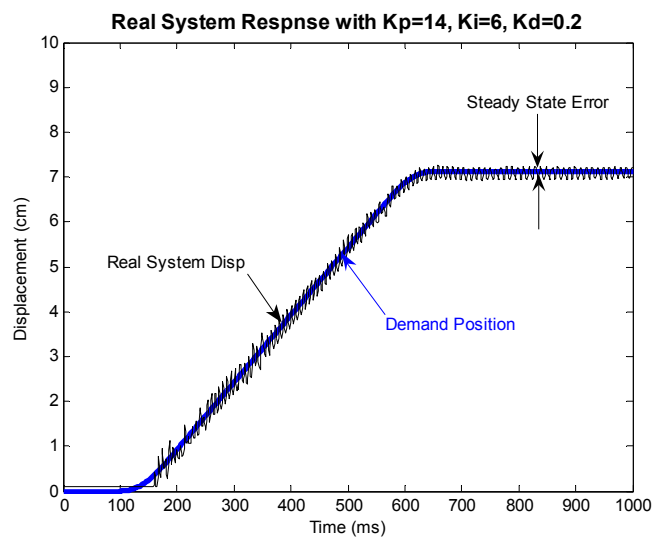

Fig.14 Variations of real and demand displacement verses time at $K p=14, K i=6$ and $K d=0.2$ 


\section{CONCLUSION}

In this paper, a method to identify and control a dynamic system is presented. The method comprises of three main stages. In the first stage, the system transfer function is identified online using HIL structure with an embedded ARMA model. The identified model is used in the second stage within SIL structure to design and optimize the controller. In the last stage, the optimized controller is applied to the real system through the HIL.

The proposed method was applied to a DC motor and a servo-pneumatic system as case studies. Simulated and experimental results showed that both systems succeeded in tracking the desired speed of a pre-specified profile with minimum error. Moreover, results showed a reasonably good match between the simulated and real system behaviors which implies that the accuracy of the system model that obtained through online identification is high. The advantages of the proposed method include accuracy, optimizing time resources and minimizing the cost, increased flexibility, and user friendly.

\section{REFERENCES}

[1] R. Isermann and N. Müller, "Design of computer controlled combustion engines", Mechatronics, Volume 13, Issue 10, Pages 10671089, December 2003.

[2] C. Lin, C. Tseng and T. Tseng, "A hardware-in-the-loop dynamics simulator for motorcycle rapid controller prototyping", Control Engineering Practice, Volume 14, Issue 12, Pages 1467-1476, December 2006.

[3] B.M. Hanson, M.C. Levesley, K. Watterson and P.G. Walker, "Hardwarein-the-loop-simulation of the cardiovascular system, with assist device testing application" Medical Engineering \& Physics, Volume 29, Issue 3, Pages 367-374, April 2007

[4] T. Kara and I. Eker. "Nonlinear modeling and identification of a DC motor for bidirectional operation with real time experiments". Energy Conversion and Management 45. pp1087-1106, 2004.

[5] T. Tutunji, M. Molhem, E. Turki "Mechatronics systems identification using an impulse response recursive algorithm." Simulation Modeling Practice and Theory 15 pp970-988, 2007.

[6] S. Abedrabbo and T. Tutunji, "Identification and analysis of hydrostatic transmission system", International Journal of Advanced Manufacturing Technology, DOI 10.1007/S00170-007-0966-3. 2007.

[7] M. T. Yan, M. H. Lee, and P. L. Yen, "Theory and Application of a combined self-tuning adaptive control and cross-coupling control in a retrofit milling machine". Mechatronics 15(2) pp193-211, 2005.

[8] T. Soderstom and P. Stoica, "System Identification", Printice Hall, 1989.

[9] K. H. Ang; G. Chong; Yun Li, "PID control system analysis, design, and technology" IEEE Transactions on Control Systems Technology, Volume 13, Issue 4, Page(s):559 - 576, July 2005. 